\title{
Krichever-Novikov Global Operator Formalism: NSR Superstring in Curved Background
}

\author{
Jnanadeva Maharana ${ }^{1}$, Samir K. Paul ${ }^{2}$, and Gautam Sengupta ${ }^{1}$ \\ 1 Institute of Physics, Bhubaneswar-751005, India \\ 2 S. N. Bose National Centre for Basic Sciences, DB17, Sector 1, Salt Lake, \\ Calcutta-700064, India
}

Received April 15, 1990; in revised form February 21, 1991

\begin{abstract}
The Neveu-Schwarz-Ramond type II closed superstring is considered to evolve in a curved space-time manifold. The Krichever-Novikov global operator formalism is used to construct the generators of a super-conformal algebra on a Riemann surface $\Sigma$. The computation for the quantum algebra of these generators is explicitly presented. It is shown that the theory is free from super-conformal anomalies if the target manifold is ten dimensional and satisfies the Ricci flatness condition.
\end{abstract}

\section{Introduction}

The string theories offer the most promising prospect of unifying all fundamental forces of nature [1]. The first quantized approach has proved to be powerful to study dynamics of string interactions. Inclusion of surfaces of non-trivial topology leads to the topological expansion which has one diagram at each order [2]. Furthermore, duality and unitarity are guaranteed at each order in this perturbation theory (a Riemann surface of a given genus is associated with each order of the perturbation expansion).

The $S$-matrix generating functional for the scattering of massless states of the string is constructed by considering the evolution of the string in the background of the corresponding massless excitations [3]. It is well known that the consistency requirements such as super-conformal invariance impose stringent constraints on the configurations of the background fields, leading to the so-called equation of motion of such fields. Indeed, the vanishing of the $\beta$-functions associated with the background fields ensures super-conformal invariance of the theory [4].

Recently, the application of some powerful mathematical results in algebraic geometry and complex analysis on Riemann surfaces have led to a detailed understanding of the multi-loop structure of string theories, in particular in the framework of operator formulation $[5,6]$. The salient feature of this formalism [5], in contrast to the path integral approach, has been a local description of 
conformal field theories over a disc cut out from a compact Riemann surface and then relating the states over the disc to states over the Riemann surface without the disc via Bogoliubov transformations. This procedure of going from a trivial topology to a nontrivial one is rather involved. We may recall the well known algebraic approach on the other hand, consists of implementing Kac-Moody and Virasoro algebras defined on the surface of a sphere, $S^{2}$, in order to describe the Hilbert space of a closed string, with punctures at $z=0$ and $z=\infty$ (the north and south poles are identified as $N$ and $S$ respectively). This approach essentially describes operator formulation on $S^{2}$ or $C P^{1}$. The Fourier modes of the fundamental fields such as bosonic and fermionic variables on the world sheet, provide the basis for defining operators acting on the Hilbert space of states. As a consequence, the modes of the energy-momentum tensor of the theory satisfy the well known Virasoro algebra on $S^{2}$.

Krichever-Novikov (KN) [7] introduced a natural extension of Virasoro algebra and also of Kac-Moody (KM) algebra on a higher genus compact Riemann surface $\Sigma$. The relation between $\mathrm{KN}$ algebra and the KM and Virasoro algebra on $S^{2}$ is explicitly shown in [8]. Subsequently there have been several attempts to study the dynamics of strings and conformal field theories in this framework [9-11]. The KN approach has been applied to the evolution of the closed bosonic string in a flat and a curved space time manifold [9], as well as NSR superstring in flat [9] and in gravitational background [10].

The purpose of this article is to envisage the evolution of the NSR closed type II superstring in a gravitational background for a Riemann surface of arbitrary genus $g$ and to implement KN operator formalism for the super-conformal algebra at the quantum level and to derive equation of motion for the background field. Recently one of us in collaboration with Fubini, Roncadelli, and Veneziano [12] has studied a type II closed superstring in curved background and has derived anomalies associated with the super-conformal symmetry on $S^{2}$. It was shown by FMRV that there exist new operator anomalies in the constraint algebra in addition to the usual $c$-number anomalies.

We may mention here that the $\mathrm{KN}$ approach has been applied to the type II superstring in curved background [10]. However in the weak field approximation, i.e. the target manifold metric is linearized around the flat space time. The weak field approximation followed in [10] is along the methods adopted by Das, Maharana, and Roy [13] for the case on $S^{2}$, where the super-conformal anomalies and critical dimension was derived from the super-Virasoro algebra.

In the present investigation, the Riemann normal coordinate expansion is adopted to expand all the generators of the super-conformal algebra and therefore they are all manifestly covariant. The vanishing of anomalies corresponds to the critical dimension ten for space time and Ricci flatness of the target manifold, therefore these are gauge invariant statements. In contrast the results in the weak field approximation are gauge dependent, i.e. the graviton satisfies the free field equation in the Lorentz gauge. Moreover, the method of Riemann normal coordinate expansion provides the procedure to compute the higher order correction in $R$ to the background equations of motion in a systematic manner. We may mention here that the super-conformal algebra for $\Sigma$ exhibits several interesting features when cast in the global $\mathrm{KN}$ operator formulation.

The rest of the paper is organised as follows. The essential features of $\mathrm{KN}$ algebra are presented in Sect. II. In Sect. III the constraint algebra for the free NSR string is obtained in the $\mathrm{KN}$ formulation. The propagation of the superstring in a 
curved background is considered in Sect. IV and the quantum $\mathrm{KN}$ algebra is explicitly presented with the anomalies. Section V contains discussions of our results and conclusions. There are two appendices containing mathematical details which are used in the main text of the paper.

\section{The Krichever-Novikov Formalism}

Krichever and Novikov [7] proposed a natural extension of the Virasoro algebra on $S^{2}$ to an arbitrary Riemann surface $\Sigma$. The Virasoro algebra being the central extension of the algebra of the meromorphic vector fields on $S^{2}, \mathrm{KN}$ generalisation of this is the central extension of the algebra of meromorphic vector fields on $\Sigma$. We recapitulate below some of the essential features of the Krichever-Novikov formalism.

The central extension of the algebra of complex valued meromorphic vector fields on $S^{2}$ is generated by the basis

is given by

$$
\left\{l_{n}(z)=z^{-n+1} \frac{\partial}{\partial z}, t\right\}
$$

$$
\left[l_{n}(z), l_{m}(z)\right]=(n-m) l_{n+m}+t \frac{n^{3}-n}{12} \delta_{n+m, 0},
$$

where $\left[l_{n}, t\right]=0$. Note that $n \in \mathbb{Z}$ and $z \in C$. The familiar example is that of the Virasoro algebra satisfied by the energy momentum tensor $T(z)$

$$
\begin{aligned}
=\sum_{n=-\infty}^{+\infty} L_{n} z^{-n}\left(\frac{d z}{z}\right)^{2}: \\
\left.\qquad L_{n}, L_{m}\right]=(n-m) L_{n+m}+\frac{c}{12}\left(n^{3}-n\right) \delta_{n+m, 0} .
\end{aligned}
$$

Krichever and Novikov provided a unique definition of the time parameter " $\tau$ " on $\Sigma$ of arbitrary genus $g \geqq 1$ and constructed bases for the space of meromorphic forms of weight $\lambda$ on $\Sigma$ with punctures at two points, $P_{ \pm}$, corresponding to $\tau \rightarrow \mp \infty$, holomorphic away from $P_{ \pm}$. This is essentially achieved through the application of Riemann-Roch theorem. Any meromorphic form of weight $\lambda$ has a generalized Laurent expansion in the corresponding basis $[7,14]$.

Let $L^{\Sigma}$ denote the algebra of meromorphic vector fields on $\Sigma$ holomorphic away from $P_{ \pm}$, and $Q$ be an arbitrary point in $\Sigma . z_{ \pm}(Q)$ are local parameters in neighbourhood of $P_{ \pm}$with $z_{ \pm}\left(P_{ \pm}\right)=0$. The Riemann-Roch theorem ensures the existence of the basis of the space of meromorphic forms of weight $\lambda$ (see 14).

We present below asymptotic forms for the basis $\left\{f_{n}^{(\lambda)}(Q)\right\}$ of the $\lambda$-forms as obtained by Krichever and Novikov for $\lambda \in \mathbb{Z}, \lambda \neq 0,1$ in the vicinity of $P_{ \pm}$; the $\lambda=0,1$ case needs special constructions. We also write asymptotic forms for the basis of these tensors for $\lambda \in \mathbb{Z}+1 / 2$. For the special case of $g=1$, we directly refer to [17].

(i) $\lambda \in \mathbb{Z}, \lambda \neq 0,1$.

$$
f_{n}^{(\lambda)}\left(z_{ \pm}\right)=\phi_{n}^{ \pm} z_{ \pm}^{ \pm n-s(\lambda)}\left[1+o\left(z_{ \pm}\right)\right](d z)^{ \pm \lambda},
$$

$\phi_{n}^{+}=1$. Here $s(\lambda)=g / 2-\lambda(g-1)$, and $n=\ldots, g / 2-1, g / 2, g / 2+1, \ldots$ Notice that the poles at $P_{ \pm}$as prescribed by $\mathrm{KN}$ are reflected in (2.3).

(ii) $\lambda=0$. 
The basis for the space of meromorphic functions holomorphic outside $P_{ \pm}$is constructed by specifying the order of the poles at $P_{ \pm}$. The basis is given by

$$
\begin{aligned}
A_{n}\left(z_{ \pm}\right) & =a_{n}^{ \pm} z_{ \pm}^{ \pm n-g / 2}\left[1+o\left(z_{ \pm}\right)\right], \quad|n| \geqq g / 2+1, a_{n}^{+}=1, \\
A_{n}\left(z_{ \pm}\right) & =a_{n}^{ \pm} z_{ \pm}^{n-g / 2 \pm 1 / 2-\varepsilon}\left[1+o\left(z_{ \pm}\right)\right], \\
|n| & \leqq g / 2, \quad n \neq g / 2, \quad a_{n}^{+}=1, \quad \varepsilon=1 / 2, \\
A_{g / 2}(Q) & =1 .
\end{aligned}
$$

Equation (2.4b) is the consequence of the Weierstrass gap theorem $[8,15]$.

(iii) $\lambda=1$.

The basis for the space of one forms is

$$
\begin{aligned}
\omega_{n}\left(z_{ \pm}\right) & =f_{-n}^{1}\left(z_{ \pm}\right) \text {given by }(2.6) \text { in the range }|n| \geqq g / 2+1 . \\
\omega_{n}\left(z_{ \pm}\right) & =\omega_{n}^{ \pm} z_{ \pm}^{\mp n+g / 2 \mp 0}\left[1+o\left(z_{ \pm}\right)\right] d z_{ \pm} \\
|n| & \leqq g / 2, \quad n \neq g / 2, \quad \omega_{n}^{+}=1 . \\
y(Q) & \equiv \omega_{g / 2}(Q)
\end{aligned}
$$

$y(Q)$ being an abelian differential of the third kind having simple poles at $P_{ \pm}$with residue \pm 1 and with periods over all cycles purely imaginary. $y(Q)$ defined through (2.6) is unique [7]. Equation (2.5) provides a basis for holomorphic differentials on $\Sigma$ (which can be written as a linear combination of the canonical basis).

(iv) $\lambda \in \mathbb{Z}+1 / 2$.

We can construct the basis, in this case, with prescribed poles at $P_{ \pm}$by taking meromorphic sections of tensor products of $\Lambda^{\lambda}$ with a given spin structure $[7,15,16]$. There are two different cases which are to be dealt with separately.

(a) Basis for the space of forms of weight $\lambda$ holomorphic outside $P_{ \pm}$and a slit from $P_{+}$to $P_{-}$along a Jordan curve (this corresponds to the Ramond sector $-\mathrm{R}$ ).

(b) Basis for the space of forms of weight $\lambda$ holomorphic outside $P_{ \pm}$(this corresponds to the Neveu-Schwarz sector - NS).

It follows from the Riemann-Roch theorem that

$$
f_{\alpha}^{(\lambda)}\left(z_{ \pm}\right)=\psi_{\alpha}^{ \pm} z_{ \pm}^{ \pm \alpha-s(\lambda)}\left[1+o\left(z_{ \pm}\right)\right]\left(d z_{ \pm}\right)^{\lambda}
$$

when $\alpha \in \mathbb{Z}$ it corresponds to the $R$-sector whereas $\alpha \in \mathbb{Z}+1 / 2$ corresponds to the NS sector. These constructions are valid for all $g$.

An explicit construction of all these bases has been given in [16]. Henceforth, we use the indices $\alpha, \beta, s, t, \ldots$ for basis when $\lambda \in \mathbb{Z}+1 / 2$ and $i, j, k, l, m, n, \ldots$ for the basis when $\lambda \in \mathbb{Z}$.

Let us now introduce the $\mathrm{KN}$ definition for "time" $\tau(Q)$ on $\Sigma . \tau(Q)$ is defined by

$$
d \tau(Q)+i d \sigma(Q)=y(Q),
$$

where $y(Q)$ is defined in (2.6). Thus,

$$
\tau(Q)=\operatorname{Re} \int_{Q_{0}}^{Q} y(Q)
$$

and

$$
\sigma(Q)=\operatorname{Im} \int_{Q_{0}}^{Q} y(Q),
$$


where $Q_{0}$ is a fixed point on $\Sigma$ except $P_{ \pm} . \Sigma$ is parametrized by one parameter family of contours: $C_{\tau}=\{\sigma(Q): \tau(Q)=$ real constant $\}$ which is the analog of " $C$ " on $S^{2} .\left\{f_{n, \alpha}^{(\lambda)}(Q)\right\}$ forms a complete set on $C_{\tau}$. We write the asymptotic form for the basis of the space of meromorphic vector fields $\left\{e_{i}(Q)\right\}$ (corresponding to $\lambda=-1$ in Eq. (2.3)) which satisfy the $g_{0}$-graded $\mathrm{KN}$ algebra on $C_{t}, g_{0}=3 g / 2$,

$$
\begin{aligned}
& e_{k}\left(z_{ \pm}\right)=a_{n}^{ \pm} z_{ \pm}^{\mp k-g_{0}+1}\left[1+o\left(z_{ \pm}\right)\right] \frac{\partial}{\partial z_{ \pm}}, \\
& k=\ldots, g / 2-1, g / 2, g / 2+1, \ldots ; a_{n}^{+}=1
\end{aligned}
$$

It follows from (2.9) that

$$
\left[e_{i}(Q), e_{j}(Q)\right]=\sum_{s=-g_{0}}^{g_{0}} C_{i j}^{s} e_{i+j-s}(Q), \quad Q \in C_{\tau},
$$

where the structure constants $C_{i j}^{s}$ are given by

$$
C_{i j}^{s}=\frac{1}{2 \pi i} \oint_{C_{\tau}}\left[e_{i}(Q), e_{j}(Q)\right] \Omega_{i+j-s}(Q)
$$

$\Omega_{n}(Q)$ are defined in Table I. Equation (2.10) is the generalization of (2.1) without central extension $(t=0)$ defined on $S^{2}$ to $\Sigma$ in the $\mathrm{KN}$ formalism. $L\left(C_{\tau}\right)$, the restriction of $L^{\Sigma}$ on $C_{\tau}$, Eq. (2.10) is isomorphic to Eq. (2.3) with $t=0$ for sufficiently large $\tau$. The central extension of $(2.10)$ is the generalized Virasoro algebra given by

$$
\left[e_{i}, e_{j}\right]=\sum_{s=-g_{0}}^{g_{0}} C_{i j}^{s} e_{i+j-s}+t \chi_{c}\left(e_{i}, e_{j}\right)
$$

Table 1. This Table refers to the global KN bases, their duality and completeness properties in terms of the meromorphic deltatensors on $\Sigma$

\begin{tabular}{rll}
\hline \multicolumn{1}{r}{} & basis & Dual basis \\
\hline-1 & $\left\{e_{i}(Q)\right\}$ & $\left\{\Omega_{j}(Q)\right\}$ \\
0 & $\left\{A_{i}(Q)\right\}$ & $\left\{\omega_{j}(Q)\right\}$ \\
$-\frac{1}{2}$ & $\left\{g_{\alpha}(Q)\right\}$ & $\left\{k_{\beta}(Q)\right\}$ \\
$+\frac{1}{2}$ & $\left\{h_{\alpha}(Q)\right\}$ & $\left\{h_{-\beta}(Q)\right\}$ \\
\hline
\end{tabular}

Duality and completeness relations

Duality

$$
\begin{aligned}
& \frac{1}{2 \pi i} \oint_{C_{\tau}} e_{i}(Q) \Omega_{j}(Q)=\delta_{i j} \\
& \frac{1}{2 \pi i} \oint_{C_{\tau}} A_{i}(Q) \omega_{j}(Q)=\delta_{i j} \\
& \frac{1}{2 \pi i} \oint_{C_{\tau}} g_{\alpha}(Q) k_{\beta}(Q)=\delta_{\alpha \beta} \\
& \frac{1}{2 \pi i} \oint_{C_{\tau}} h_{\alpha}(Q) h_{-\beta}(Q)=\delta_{\alpha \beta}
\end{aligned}
$$

Completeness

$$
\begin{aligned}
& \frac{1}{2 \pi i} \sum e_{i}(Q) \Omega_{i}\left(Q^{\prime}\right)=D_{\tau}\left(Q, Q^{\prime}\right) \\
& \frac{1}{2 \pi i} \sum A_{i}(Q) \omega_{i}\left(Q^{\prime}\right)=\Delta_{\tau}\left(Q, Q^{\prime}\right) \\
& \frac{1}{2 \pi i} \sum g_{\alpha}(Q) k_{\alpha}\left(Q^{\prime}\right)=d_{\tau}\left(Q, Q^{\prime}\right) \\
& \frac{1}{2 \pi i} \sum h_{\alpha}(Q) h_{-\alpha}\left(Q^{\prime}\right)=\delta_{\tau}\left(Q, Q^{\prime}\right)
\end{aligned}
$$


with $\left[e_{i}, t\right]=0$; here $t$ is a central element and

$$
\chi_{c}\left(e_{i}, e_{j}\right)=\frac{1}{24 \pi i} \oint_{C_{\tau}} \tilde{\chi}\left(e_{i}, e_{j}\right) .
$$

$C$ is any contour on $\Sigma$ not passing through $P_{ \pm}$and $\tilde{X}(f, g)=f^{\prime \prime \prime}(z) g(z) d z$, where $f(z) \frac{\partial}{\partial z}$ and $g(z) \frac{\partial}{\partial z}$ are representations of two vector fields in an admissible coordinate system. Equations (2.12) and (2.13) give all central extensions of $L^{\Sigma}$ by standard calculations of two dimensional cohomology of algebras [7]. This gives generalization of $L$ given by (2.1) on $S^{2}$ to $\Sigma$. The properties of the cocycles can be found in [7].

Let $\left\{g_{\alpha}(Q)\right\}$ denote the basis for the space of meromorphic $-1 / 2$ forms corresponding to $\lambda=-1 / 2$ in (2.7). Then the commutator of the vector fields with $g_{\alpha}(Q)$ is

$$
\left[e_{i}(Q), g_{\alpha}(Q)\right]=\sum_{s=-g_{0}}^{g_{0}} H_{i \alpha}^{s} g_{i+\alpha-s}(Q), \quad Q \in C_{\tau},
$$

where the structure constants $H_{i \alpha}^{s}$ are given by

$$
H_{i j}^{s}=\frac{1}{2 \pi i} \oint_{C_{\tau}}\left[e_{i}(Q), g_{\alpha}(Q)\right] y_{i+\alpha-s}(Q)
$$

$y_{\beta}(Q)$ 's are given in Table I. Finally we write the central extension of the algebra of $-1 / 2$ forms in order to obtain the super $\mathrm{KN}$ algebra,

$$
\left\{g_{\alpha}(Q), g_{\beta}(Q)\right\}=\sum_{p=-g}^{g} B_{\alpha \beta}^{p} e_{\alpha+\beta-p / 2}(Q), \quad Q \in C_{\tau} .
$$

The structure constants $B_{\alpha \beta}^{p}$ are given by

$$
B_{\alpha \beta}^{p}=\frac{1}{2 \pi i} \oint_{C_{\tau}}\left\{g_{\alpha}(Q), g_{\beta}(Q)\right\} \Omega_{\alpha+\beta-p / 2}(Q) .
$$

Here (2.16) is the algebra of meromorphic $-1 / 2$ forms. The central extension of (2.16) is given by

$$
\left\{g_{\alpha}, g_{\beta}\right\}=\sum_{p=-g}^{g} B_{\alpha \beta}^{p} e_{\alpha+\beta-p / 2}+t \phi_{c}\left(g_{\alpha}, g_{\beta}\right)
$$

with the requirement that $\left[g_{\alpha}, t\right]=0$. The two cocycle $\phi_{c}\left(g_{\alpha}, g_{\beta}\right)$ are given by

$$
\phi_{c}\left(g_{\alpha}, g_{\beta}\right)=\frac{1}{6 \pi i} \oint_{c_{\tau}} \tilde{\phi}\left(g_{\alpha}, g_{\beta}\right)
$$

$\tilde{\phi}(\varrho, \sigma)=\varrho^{\prime}(z) \sigma^{\prime}(z) d z$, where $\varrho(z)(d z)^{-1 / 2}$ and $\sigma(z)(d z)^{-1 / 2}$ are representations of two $-1 / 2$ forms in an admissible coordinate system. The properties of the cocycles defined above, Eqs. (2.13) and (2.19) are given in the paper of Bonora, Martellini, Rinaldi, and Russo [9].

Equations (2.12), (2.14), and (2.18) comprise the centrally extended super-KN algebra which is the generalization of the super-Virasoro algebra. Finally, we set the notations for the basis of some of forms of weight $\lambda$ and the dual basis of forms of weight $1-\lambda$. We also give the duality relations and completeness relations in Table I. All points $Q, Q^{\prime}$ in the same row belong to the same $C_{\tau}$. Notice that $\left\{\bar{f}_{n, \alpha}^{(\lambda)}(Q)\right.$, $\left.Q \in C_{\tau}\right\}$ forms an alternative basis with asymptotic forms (2.3)-(2.7) with $z_{ \pm}$ replaced by $\bar{z}_{ \pm}$. This basis forms the antiholomorphic analog of what has been 
described. The duality and completeness relations hold for the barred dual basis with negative signs in front of the contour integrals and the summations, as shown in Table 1.

In the next section we construct a quantum constraint algebra for NSR superstring in flat as well as curved space-time manifold in which the Fourier modes of the operators provide a realization of the centrally extended KricheverNovikov algebras given by (2.12), (2.14), and (2.18).

\section{Neveu-Schwarz-Ramond String in Flat Space-Time}

This section deals with the generalization of the superconformal algebra, associated with the NSR string, to the Riemann surface, $\Sigma$, of arbitrary genus in the KN framework. The close correspondence of the KN approach with the operator formalism on $S^{2}$ renders such a generalization (to $\Sigma$ ) to be both elegant and simple. Moreover, this is a prelude to our subsequent investigation of the NSR superstring in a curved background space-time manifold. We present the canonical Poisson brackets for the NSR string on $\Sigma$ and derive the classical constraint algebra. Next, we canonically quantize the model on $\Sigma$ and derive the quantum algebra of the normal ordered superconformal generators.

The gauge fixed action (in ON gauge) for the free NSR superstring is

$$
S=\frac{1}{2} \int d \sigma d \tau\left[\partial_{a} X^{\mu} \partial^{a} X_{\mu}+i \bar{\chi}^{\mu} \gamma^{a} \partial_{a} \chi_{\mu}\right]
$$

Here $\tau$ and $\sigma$ parametrize the world sheet. $\chi^{\mu}$ are the Majorana spinors, superpartner of the bosonic coordinates $X^{\mu}$. The two dimensional Dirac matrices are defined as $\gamma^{0}=\sigma_{2}, \gamma^{1}=i \sigma_{1}$, and $\gamma_{5}=\gamma^{0} \gamma^{1}=\sigma_{3}$. We denote the world sheet labels by $a, b, \ldots$ here and the space-time indices by $\mu, v, \ldots$; all target manifold tensors are raised and lowered by the flat metric, $\eta_{\mu \nu}$, in this section. The generators of the superconformal transformations on $S^{2}$ are

$$
\begin{gathered}
T_{ \pm}^{0}(\sigma, \tau)=P_{ \pm}^{\mu}(\sigma, \tau) P_{ \pm \mu}(\sigma, \tau) \pm \frac{i}{2} \psi_{ \pm}^{\mu}(\sigma, \tau) \psi_{ \pm \mu}^{\prime}(\sigma, \tau), \\
J_{ \pm}^{0}(\sigma, \tau)=2 P_{ \pm}^{\mu}(\sigma, \tau) \psi_{ \pm \mu}(\sigma, \tau) .
\end{gathered}
$$

Here $T_{ \pm}^{0}$ and $J_{ \pm}^{0}$ are the energy momentum tensor and the supercharge densities respectively,

$$
P_{ \pm \mu}=\frac{1}{2}\left(P_{\mu} \pm X_{\mu}^{\prime}\right)
$$

are the Fubini-Veneziano fields, $P_{\mu}$ is the momentum conjugate to $X^{\mu}$ and the world sheet chiral fermions are defined through the equation

$$
\psi_{ \pm}^{\mu}=\frac{1}{2}\left(1 \mp \gamma_{5}\right) \chi^{\mu} .
$$

The theory is defined over the Riemann surface, $\Sigma$, as follows; we Wick rotate $\sigma \rightarrow i \sigma(0 \leqq \sigma \leqq 2 \pi,-\infty \leqq \tau \leqq=+\infty)$. At a generic point $Q$ on $\Sigma$, we can write a local coordinate system $W: W(Q)=\tau(Q)+i \sigma(Q)$. Now the costraints $(3.2)$ are generalized to $\Sigma$ as

$$
\begin{aligned}
& T_{ \pm}^{0}(Q)=P_{ \pm}^{\mu}(Q) P_{ \pm \mu}(Q) \pm \frac{i}{2} \psi_{ \pm}^{\mu}(Q) d_{Q} \psi_{ \pm \mu}(Q), \\
& J_{ \pm}^{0}(Q)=2 P_{ \pm}^{\mu}(Q) \psi_{ \pm \mu}(Q),
\end{aligned}
$$

with

$$
P_{ \pm}^{\mu}(Q)=\frac{1}{2}\left(P_{\mu} \pm d_{Q} X^{\mu}(Q)\right) \text {. }
$$


Here $d_{Q}$ denotes the global derivative on $\Sigma$ having a local expression $d W \frac{\partial}{\partial W}$. Note that $T_{+}^{0}, J_{+}^{0}$ and $T_{-}^{0}, J_{-}^{0}$ are the analog of the holomorphic and antiholomorphic generators on $S^{2}$ respectively.

The relevant operators $X^{\mu}, P_{\mu}, P_{ \pm}^{\mu}$, and $\psi_{ \pm}^{\mu}$ that appear in the definition of the generators (3.5) can be globally Laurent expanded in the appropriate $\mathrm{KN}$ bases (refer to Table 1),

where

$$
\begin{gathered}
X^{\mu}(Q)=\sum_{n} X_{n}^{\mu} A_{n}(Q), \\
P_{\mu}(Q)=\sum_{n} P_{n \mu} \omega_{n}(Q), \\
P_{+}^{\mu}(Q)=\sum_{n} \alpha_{n}^{\mu} \omega_{n}(Q), \\
P_{-}^{\mu}(Q)=\sum_{n} \bar{\alpha}_{n}^{\mu} \bar{\omega}_{n}(Q), \\
\psi_{+}^{\mu}(Q)=\sum_{s} \psi_{+s}^{\mu} h_{s}(Q), \\
\psi_{-}^{\mu}(Q)=\sum_{s} \psi_{-s}^{\mu}(Q) \bar{h}_{s}(Q),
\end{gathered}
$$

and

$$
\begin{aligned}
& \alpha_{n}^{\mu}=P_{n}^{\mu}+\sum_{l} X_{l}^{\mu} \gamma_{l n}, \\
& \bar{\alpha}_{n}^{\mu}=\bar{P}_{n}^{\mu}-\sum_{l} \bar{X}_{l}^{\mu} \bar{\gamma}_{l n},
\end{aligned}
$$

$$
\gamma_{l n}=\frac{1}{2 \pi i} \oint_{C_{\tau}} d A_{l}(Q) A_{n}(Q)
$$

The canonical Poisson brackets on $\Sigma$ are defined on an equal time curve $C_{\tau}$

$$
\begin{gathered}
\left\{X^{\mu}(Q), P^{v}\left(Q^{\prime}\right)\right\}=\eta^{\mu v} \Delta_{\tau}\left(Q, Q^{\prime}\right), \\
\left\{\psi_{ \pm}^{\mu}(Q), \psi_{ \pm}^{v}\left(Q^{\prime}\right)\right\}=-i \eta^{\mu v} \delta_{\tau}\left(Q, Q^{\prime}\right),
\end{gathered}
$$

and all other Poisson brackets among string coordinates vanish.

Now we are in a position to compute the classical Poisson bracket algebra on $C_{\tau}$, and present below the algebra involving $J_{+}^{0}$ and $T_{+}^{0}$ explicitly,

$$
\begin{aligned}
& \left\{J_{+}^{0}(Q), J_{+}^{0}\left(Q^{\prime}\right)\right\} \\
& =4\left[P_{+}^{\mu}(Q) P_{+\mu}\left(Q^{\prime}\right) \delta_{\tau}\left(Q, Q^{\prime}\right)-\frac{1}{4 \pi} \psi_{+}^{\mu}(Q) \psi_{+\mu}\left(Q^{\prime}\right) \sum_{n, m} \gamma_{n m} \omega_{n}(Q) \omega_{m}\left(Q^{\prime}\right)\right] \\
& \left\{T_{+}^{0}(Q), J_{+}^{0}\left(Q^{\prime}\right)\right\} \\
& =i\left[P_{+}^{\mu}(Q) \psi_{+}^{\mu}\left(Q^{\prime}\right)\left(d_{Q^{\prime}} \Delta_{\tau}\left(Q, Q^{\prime}\right)-d_{Q^{\prime}} \Delta_{\tau}\left(Q^{\prime}, Q\right)\right)\right. \\
& \left.+P_{+}^{\mu}\left(Q^{\prime}\right) \psi_{+\mu}\left(Q^{\prime}\right) d_{Q^{\prime}} \delta_{\tau}\left(Q, Q^{\prime}\right)-P_{+}^{\mu}\left(Q^{\prime}\right) d_{Q} \psi_{+\mu}(Q) \delta_{\tau}\left(Q, Q^{\prime}\right)\right] \\
& \left\{T_{+}^{0}(Q), T_{+}^{0}\left(Q^{\prime}\right)\right\}=-\frac{1}{\pi} P_{+}^{\mu}(Q) P_{+\mu}\left(Q^{\prime}\right) \sum_{n, m} \gamma_{n m} \omega_{n}(Q) \omega_{m}\left(Q^{\prime}\right) \\
& +\frac{1}{4}\left[\psi_{+}^{\mu}(Q) \psi_{+\mu}\left(Q^{\prime}\right) d_{Q} d_{Q^{\prime}} \delta_{\tau}\left(Q, Q^{\prime}\right)\right. \\
& -\psi_{+}^{\mu}(Q) d_{Q^{\prime}} \psi_{+\mu}\left(Q^{\prime}\right) d_{Q^{\prime}} \delta_{\tau}\left(Q, Q^{\prime}\right) \\
& -d_{Q} \psi_{+}^{\mu}(Q) \psi_{+\mu}\left(Q^{\prime}\right) d_{Q^{\prime}} \delta_{\tau}\left(Q, Q^{\prime}\right) \\
& \left.+d_{Q} \psi_{+}^{\mu}(Q) d_{Q^{\prime}} \psi_{+\mu}\left(Q^{\prime}\right) \delta_{\tau}\left(Q, Q^{\prime}\right)\right] \\
& \left\{T_{+}^{0}(Q), J_{-}^{0}\left(Q^{\prime}\right)\right\}=\left\{T_{+}^{0}(Q), T_{-}^{0}\left(Q^{\prime}\right)\right\}=\left\{J_{+}^{0}(Q), J_{-}^{0}\left(Q^{\prime}\right)\right\}=0
\end{aligned}
$$


The algebra involving $T_{-}^{0}$ and $J_{-}^{0}$ can be computed in a straightforward manner.

We recall that the classical Virasoro algebra on $S^{2}$ has the following form (the equal $\tau$ Poisson bracket algebra):

$$
\begin{gathered}
\left\{J_{+}^{0}(\sigma), J_{+}^{0}\left(\sigma^{\prime}\right)\right\}=2\left[T_{+}^{0}(\sigma)+T_{+}^{0}\left(\sigma^{\prime}\right)\right] \delta\left(\sigma-\sigma^{\prime}\right), \\
\left\{T_{+}^{0}(\sigma), J_{+}^{0}\left(\sigma^{\prime}\right)\right\}=\frac{i}{2}\left[2 J_{+}^{0}(\sigma)+J_{+}^{0}\left(\sigma^{\prime}\right)\right] \frac{\partial}{\partial \sigma} \delta\left(\sigma-\sigma^{\prime}\right), \\
\left\{T_{+}^{0}(\sigma), T_{+}^{0}\left(\sigma^{\prime}\right)\right\}=i\left[T_{+}^{0}(\sigma)+T_{+}^{0}\left(\sigma^{\prime}\right)\right] \frac{\partial}{\partial \sigma} \delta\left(\sigma-\sigma^{\prime}\right), \\
\left\{T_{+}^{0}(\sigma), J_{-}^{0}\left(\sigma^{\prime}\right)\right\}=\left\{T_{+}^{0}(\sigma), T_{-}^{0}\left(\sigma^{\prime}\right)\right\}=\left\{J_{+}^{0}(\sigma), J_{-}^{0}\left(\sigma^{\prime}\right)\right\}=0 .
\end{gathered}
$$

We notice that the algebra on $S^{2}$ neatly factorizes, i.e. the generators on the right-hand side are either a function of $\sigma$ or that of $\sigma^{\prime}$ and they are multiplied by a $\delta$-function or a derivative of a $\delta$-function. There is no such factorization in Eqs. (3.16)-(3.18) due to the use of generic global coordinates $Q$ and $Q^{\prime}$ in the algebra of the generators. The algebra on $S^{2}$, as in (3.20), is obtained through certain manipulations of the $\delta$-functions and their derivatives. The bilocal expressions in $Q$ and $Q^{\prime}$ appear on the right-hand side of (3.16)-(3.18) due to the presence of the global meromorphic delta-tensors which do not possess all the attributes of the ordinary $\delta$-functions defined on $S^{2}$ (ref. to Table 1). However, when we restrict the algebra (3.16)-(3.18) on equal time circles in a local complex coordinate system $W$ around $P_{ \pm}$, we recover the superconformal algebra over $S^{2}$. Therefore we may conclude that expressions (3.16)-(3.19) are, indeed, the proper generalization of the classical super-Virasoro algebra on $S^{2}$ to the analogous algebra on an arbitrary Riemann surface, $\Sigma$.

The theory is canonically quantized by replacing the canonical Poisson brackets by the corresponding commutators or anticommutators on an equal time curve $C_{\tau}$ on $\Sigma$,

$$
\begin{aligned}
& {\left[X^{\mu}(Q), P^{v}\left(Q^{\prime}\right)\right]=i \eta^{\mu \nu} \Delta_{\tau}\left(Q, Q^{\prime}\right),} \\
& \left\{\psi_{ \pm}^{\mu}(Q), \psi_{ \pm}^{v}\left(Q^{\prime}\right)\right\}=\eta^{\mu v} \delta_{\tau}\left(Q, Q^{\prime}\right) .
\end{aligned}
$$

The operators appearing in various mode expansions (3.6)-(3.13) satisfy the quantum bracket relations,

$$
\begin{gathered}
{\left[X_{n}^{\mu}, P_{m}^{v}\right]=\frac{1}{2 \pi} \eta^{\mu v} \delta_{n, m},} \\
{\left[\bar{X}_{n}^{\mu}, \bar{P}_{m}^{v}\right]=-\frac{1}{2 \pi} \eta^{\mu v} \delta_{n, m},} \\
\left\{\psi_{+s}^{\mu}, \psi_{+s^{\prime}}^{v}\right\}=\frac{1}{2 \pi i} \eta^{\mu v} \delta_{s,-s^{\prime}}, \\
\left\{\psi_{-s}^{\mu}, \psi_{-s^{\prime}}^{v}\right\}=-\frac{1}{2 \pi i} \eta^{\mu \nu} \delta_{s,-s}, \\
{\left[\alpha_{n}^{\mu}, \alpha_{m}^{v}\right]=-\frac{1}{2 \pi} \gamma_{n m},}
\end{gathered}
$$




$$
\begin{gathered}
{\left[\bar{\alpha}_{n}^{\mu}, \bar{\alpha}_{m}^{v}\right]=-\frac{1}{2 \pi} \bar{\gamma}_{n m},} \\
{\left[X_{n}^{\mu}, \alpha_{m}^{v}\right]=\frac{1}{2 \pi} \eta^{\mu v} \delta_{n, m},} \\
{\left[\bar{X}_{n}^{\mu}, \bar{\alpha}_{m}^{v}\right]=-\frac{1}{2 \pi} \eta^{\mu v} \delta_{n, m} .}
\end{gathered}
$$

In the quantized theory, we have to define operators which are properly normal ordered. Krichever and Novikov have discussed this issue in detail for the case of global operator formalism. It was noted in Sect. II that as $Q \rightarrow P_{ \pm}, \tau \rightarrow \mp \infty$ on $\Sigma$. Therefore, we can identify the limits $Q \rightarrow P_{+}$with $|0\rangle_{\text {in }}$ and $Q \rightarrow P_{-}$with $_{\text {out }}\langle 0|$. Then if we demand regular behaviour of the correlators, the following expressions must be finite:

$$
\begin{aligned}
& \operatorname{Lim}_{Q \rightarrow P_{+}} X^{\mu}(Q)|0\rangle_{\text {in }}=\text { finite }, \\
& \operatorname{Lim}_{Q \rightarrow P_{-}}{ }_{\text {out }}\langle 0| X^{\mu}(Q)=\text { finite. }
\end{aligned}
$$

Using the asymptotic forms for the bases at $P_{ \pm}$Eqs. (2.3)-(2.4) and the expansions for $X^{\mu}(Q)$ in (3.6), we conclude

$$
\begin{aligned}
& \operatorname{Lim}_{z_{+} \rightarrow 0} X^{\mu}\left(z_{+}\right)|0\rangle=\text { finite }, \\
& \operatorname{Lim}_{z_{-} \rightarrow 0}\langle 0| X^{\mu}\left(z_{-}\right)=\text {finite. }
\end{aligned}
$$

So that

$$
\begin{gathered}
X_{n}^{\mu}|0\rangle=0, \quad n<g / 2, \\
\langle 0| X_{n}^{\mu}=0, \quad n \geqq-g / 2 .
\end{gathered}
$$

Similarly, for the momentum operator we obtain

$$
\begin{gathered}
P_{n}^{\mu}|0\rangle=0, \quad n \geqq g / 2, \\
\langle 0| P_{n}^{\mu}=0, \quad n<-g / 2 .
\end{gathered}
$$

Notice that there is a region of overlap in the $X_{n}^{\mu}$ ordering and consequently the modes in this overlap region annihilate both the "in" and the "out" vacua. Thus, the vacuum has vanishing norm giving rise to inconsistencies. This problem could be resolved by adopting the concept of universal vacuum introduced in [16] and this vacuum possesses unit norm, i.e. $(0 \mid 0)=1$.

Now the operation of the modes is defined to be

$$
\begin{gathered}
\left.X_{n}^{v} \mid 0\right)=0, \quad n<-g / 2, \\
\left(0 \mid X_{n}^{v}=0, \quad n \geqq g / 2,\right. \\
\left.P_{n} \mid 0\right)=0, \quad n \geqq g / 2, \\
\left(0 \mid P_{n}^{v}=0, \quad n<-g / 2 .\right.
\end{gathered}
$$

We remark that there is exactly " $g$ " $P_{n}$ 's which do not annihilate the left or the right vacuum. This is also the dimension of holomorphic 1-forms on $\Sigma$ Eqs. (2.5)-(2.6). 
For the modes of the Fubini-Veneziano fields $P_{ \pm}^{\mu}(Q)$, the corresponding relations are

$$
\begin{gathered}
\left.\alpha_{n}^{\mu} \mid 0\right)=0, \quad n \geqq g / 2, \\
\left(0 \mid \alpha_{n}^{\mu}=0, \quad n<-g / 2 .\right.
\end{gathered}
$$

In the interval $I \equiv(-g / 2 \leqq n<g / 2)$ the $\alpha$ operators do not commute since $\gamma_{n m} \neq 0$. Therefore, there is considerable amount of freedom in the choice of the normal ordering conventions. The general prescription of $\mathrm{KN}$ [7] may be suitably utilized for our purpose as follows:

$$
: \alpha_{n}^{\mu} \alpha_{m}^{v}:=\left\{\begin{array}{llll}
\alpha_{n}^{\mu} \alpha_{m}^{v} & m \geqq g / 2 & \text { or } & n<-g / 2 \\
\alpha_{m}^{v} \alpha_{n}^{\mu} & n \geqq g / 2 & \text { or } & m<-g / 2
\end{array} .\right.
$$

This may be generalized to include the interval $I$,

$$
: \alpha_{n}^{\mu} \alpha_{m}^{v}:=\alpha_{n}^{\mu} \alpha_{m}^{v}+\frac{\eta^{\mu \nu}}{2 \pi} \tilde{\gamma}_{n m}
$$

where

$$
\tilde{\gamma}_{n m}=\left\{\begin{array}{lllll}
0 & \text { for } & m \geqq g / 2 & \text { or } & n<-g / 2 \\
\gamma_{n m} & \text { for } & n \geqq g / 2 & \text { or } & m<-g / 2
\end{array} .\right.
$$

In the case of the fermionic fields the normal ordering prescription is unique:

$$
\begin{array}{ll}
\left.\psi_{ \pm}^{\mu} \mid 0\right)=0, & s<0, \\
\left(0 \mid \psi_{ \pm}^{\mu}=0,\right. & s>0 .
\end{array}
$$

We need correlators of various fields in order to compute the quantum superconformal algebra. The relevant correlators are given below:

$$
\begin{gathered}
\left\langle P_{+}^{\mu}(Q) X^{\nu}\left(Q^{\prime}\right)\right\rangle=-\frac{i}{2} \eta^{\mu \nu} \Delta_{\tau}^{+}\left(Q^{\prime}, Q\right), \\
\left\langle X^{\nu}\left(Q^{\prime}\right) P_{+}^{\mu}(Q)\right\rangle=\frac{i}{2} \eta^{\mu \nu} \Delta_{\tau}^{-}\left(Q^{\prime}, Q\right), \\
\left\langle P_{-}^{\mu}(Q) X^{\nu}\left(Q^{\prime}\right)\right\rangle=-\frac{i}{2} \eta^{\mu \nu} \bar{\Delta}_{\tau}^{+}\left(Q^{\prime}, Q\right), \\
\left\langle X^{\nu}\left(Q^{\prime}\right) P_{-}^{\mu}(Q)\right\rangle=\frac{i}{2} \eta^{\mu \nu} \bar{\Delta}_{\tau}^{-}\left(Q^{\prime}, Q\right), \\
\left\langle\psi_{+}^{\alpha}\left(Q^{\prime}\right) \psi_{+}^{\beta}(Q)\right\rangle=\eta^{\alpha \beta} \delta_{\tau}^{-}\left(Q^{\prime}, Q\right), \\
\left\langle\psi_{-}^{\alpha}\left(Q^{\prime}\right) \psi_{-}^{\beta}(Q)\right\rangle=\eta^{\alpha \beta} \bar{\delta}_{\tau}^{-}\left(Q^{\prime}, Q\right),
\end{gathered}
$$

where

and

$$
\begin{aligned}
& \Delta_{\tau}^{+}\left(Q^{\prime}, Q\right)=\frac{1}{2 \pi i} \sum_{n \geqq g / 2} A_{n}\left(Q^{\prime}\right) \omega_{n}(Q), \\
& \delta_{\tau}^{+}\left(Q^{\prime}, Q\right)=\frac{1}{2 \pi i} \sum_{s>0} h_{s}\left(Q^{\prime}\right) h_{-s}(Q),
\end{aligned}
$$

$$
\begin{aligned}
& \Delta_{\tau}=\Delta_{\tau}^{+}+\Delta_{\tau}^{-}, \\
& \delta_{\tau}=\delta_{\tau}^{+}+\delta_{\tau}^{-} .
\end{aligned}
$$


The tensors in the conjugate basis are $\bar{\Delta}^{ \pm}$and $\bar{\delta}^{ \pm}$and they are exact analogs of complex conjugates. We also have the following correlators:

$$
\begin{aligned}
& \left\langle P_{+}^{\mu}(Q) P_{+}^{v}\left(Q^{\prime}\right)\right\rangle=-\frac{\eta^{\mu \nu}}{4 \pi} \sum_{n, m} \omega_{n}(Q) \omega_{m}\left(Q^{\prime}\right) \tilde{\gamma}_{n m}, \\
& \left\langle P_{-}^{\mu}(Q) P_{-}^{v}\left(Q^{\prime}\right)\right\rangle=-\frac{\eta^{\mu \nu}}{4 \pi} \sum_{n, m} \bar{\omega}_{n}(Q) \bar{\omega}_{m}\left(Q^{\prime}\right) \overline{\tilde{\gamma}}_{n m}, \\
& \left\langle P_{+}^{\mu}(Q) P_{-}^{v}\left(Q^{\prime}\right)\right\rangle=\frac{1}{4} \sum_{n, m} \omega_{n}(Q) \bar{\omega}_{m}\left(Q^{\prime}\right)\left\langle\alpha_{n}^{\mu} \bar{\alpha}_{m}^{v}\right\rangle .
\end{aligned}
$$

Now we are in a position to compute the quantum algebra for the normal ordered operators. The algebra for $: T_{+}^{0}:$ and $: J_{+}^{0}:$ are presented explicitly including the anomaly terms.

$$
\begin{aligned}
& \left\{: J_{+}^{0}(Q):,: J_{+}^{0}\left(Q^{\prime}\right):\right\}=4\left[: P_{+}^{\mu}(Q) P_{+\mu}\left(Q^{\prime}\right): \delta_{\tau}\left(Q, Q^{\prime}\right)\right. \\
& \left.-\frac{1}{4 \pi}: \psi_{+}^{\mu}(Q) \psi_{+\mu}\left(Q^{\prime}\right): \sum_{n, m} \gamma_{n m} \omega_{n}(Q) \omega_{m}\left(Q^{\prime}\right)\right] \\
& -\frac{D}{\pi}\left[\delta_{\tau}^{+}\left(Q, Q^{\prime}\right) \sum_{n, m} \omega_{n}(Q) \omega_{m}\left(Q^{\prime}\right) \tilde{\gamma}_{n m}\right. \\
& \left.+\delta_{\tau}^{-}\left(Q, Q^{\prime}\right) \sum_{n, m} \omega_{n}(Q) \omega_{m}\left(Q^{\prime}\right) \tilde{\gamma}_{n m}\right] \\
& {\left[: T_{+}^{0}(Q):,: J_{+}^{0}\left(Q^{\prime}\right):\right]=i\left[: P_{+}^{\mu}(Q) P_{+\mu}\left(Q^{\prime}\right):\left(d_{Q} \Delta_{\tau}\left(Q, Q^{\prime}\right)-d_{Q^{\prime}} \Delta_{\tau}\left(Q^{\prime}, Q\right)\right)\right.} \\
& \left.+: P_{+}^{\mu}(Q) \psi_{+\mu}\left(Q^{\prime}\right): d_{Q} \delta_{\tau}\left(Q, Q^{\prime}\right)-: P_{+}^{\mu}\left(Q^{\prime}\right) d_{Q} \psi_{+\mu}(Q): \delta_{\tau}\left(Q, Q^{\prime}\right)\right], \\
& {\left[: T_{+}^{0}(Q):,: T_{+}^{0}\left(Q^{\prime}\right):\right]=-\frac{1}{\pi}: P_{+}^{\mu}(Q) P_{+\mu}\left(Q^{\prime}\right): \sum_{n, m} \gamma_{n m} \omega_{n}(Q) \omega_{m}\left(Q^{\prime}\right)} \\
& +\frac{1}{4}\left[: \psi_{+}^{\mu}(Q) \psi_{+\mu}\left(Q^{\prime}\right): d_{Q} d_{Q^{\prime}} \delta_{\tau}\left(Q, Q^{\prime}\right)\right. \\
& -: \psi_{+}^{\mu}(Q) d_{Q^{\prime}} \psi_{+\mu}\left(Q^{\prime}\right): d_{Q^{\prime}} \delta_{\tau}\left(Q, Q^{\prime}\right) \\
& -: d_{Q} \psi_{+}^{\mu}(Q) \psi_{+\mu}\left(Q^{\prime}\right): d_{Q^{\prime}} \delta_{\tau}\left(Q, Q^{\prime}\right) \\
& \left.+: d_{Q} \psi_{+}^{\mu}(Q) d_{Q^{\prime}} \psi_{+\mu}\left(Q^{\prime}\right): \delta_{\tau}\left(Q, Q^{\prime}\right)\right] \\
& +\frac{D}{4 \pi^{2}}\left[\frac{1}{2} \sum_{n, m, k, s} \gamma_{n m} \gamma_{k s} \omega_{n}(Q) \omega_{m}\left(Q^{\prime}\right) \omega_{k}(Q) \omega_{s}\left(Q^{\prime}\right)\right. \\
& \left.+\sum_{n, m, k, s} \gamma_{n m} \tilde{\gamma}_{k s} \omega_{n}(Q) \omega_{m}\left(Q^{\prime}\right) \omega_{k}\left(Q^{\prime}\right) \omega_{s}(Q)\right] \\
& +\frac{D}{4}\left[\delta_{\tau}^{+}\left(Q^{\prime}, Q\right) d_{Q} d_{Q^{\prime}} \delta_{\tau}^{+}\left(Q, Q^{\prime}\right)\right. \\
& -\delta_{\tau}^{-}\left(Q^{\prime}, Q\right) d_{Q^{\prime}} d_{Q^{\prime}} \delta_{\tau}^{-}\left(Q^{\prime}, Q\right) \\
& -d_{Q^{\prime}} \delta_{\tau}^{+}\left(Q^{\prime}, Q\right) d_{Q^{\prime}} \delta_{\tau}^{+}\left(Q^{\prime}, Q\right) \\
& \left.+d_{Q^{\prime}} \delta_{\tau}^{-}\left(Q^{\prime}, Q\right) d_{Q^{\prime}} \delta_{\tau}^{-}\left(Q^{\prime}, Q\right)\right] \\
& {\left[: T_{+}^{0}(Q):,: T_{-}^{0}\left(Q^{\prime}\right):\right]=\left[: T_{+}^{0}(Q):,: J_{-}^{0}\left(Q^{\prime}\right):\right]=\left\{: J_{+}^{0}(Q):,: J_{-}^{0}\left(Q^{\prime}\right):\right\}=0 \text {. }}
\end{aligned}
$$


Notice that, in contrast to the classical algebra (3.16)-(3.19), the right-hand side of (3.55) - (3.58) are normal ordered, and additional $c$-number terms appear in Eqs. (3.55) and (3.57). These are the central extensions of the superconformal algebra. We remark here that the restriction of the algebra (3.55)-(3.58) to a local coordinate system reproduces the centrally extended algebra on $S^{2}$ (see Appendix A).

The presence of the $c$-number terms in (3.55) and (3.57) renders the superconformal algebra anomalous at the quantum level. We recall that the action (3.1) is ON gauge fixed; therefore, we have to take into consideration the superconformal ghost contributions to the action. The superghost energy momentum tensor algebra is presented in Appendix B. We show that the anomaly in energy momentum tensor algebra is cancelled by the contributions from the corresponding ghost algebra for space-time dimension, $D=10$. This concludes our discussion of the free NSR string.

\section{Superstring in Curved Background}

In this section, we derive the quantum constraint algebra for the propagation of the NSR superstring in a curved space-time manifold with background metric, $g_{\mu \nu}(X)$, on $\Sigma$ in the KN framework.

The orthonormal gauge-fixed action is

$$
\begin{aligned}
S= & \int d \tau d \sigma\left[\frac{1}{2} g_{\mu \nu}(X) \partial_{a} X^{\mu} \partial^{a} X^{v}+\frac{i}{2} \bar{\chi}^{\mu} \gamma^{a} D_{a} \chi^{v} g_{\mu \nu}(X)\right. \\
& \left.+\frac{1}{12} R_{\mu \nu \varrho \kappa}\left(\bar{\chi}^{\mu} \chi^{\varrho}\right)\left(\bar{\chi}^{v} \chi^{\kappa}\right)\right],
\end{aligned}
$$

where the covariant derivative $D_{a}$ stands for

$$
D_{a} \equiv \partial_{a} \chi^{\alpha}-\omega_{\mu, \alpha \beta} \chi^{\beta} \partial X \mu \text {. }
$$

$R_{\mu \nu \varrho \kappa}$ is the Riemann-Christoffel tensor in the target space. The target space vielbeins are related to the metric by the relation

$$
g_{\mu \nu}(X)=e_{\mu \alpha}(X) e_{\nu}^{\alpha}(X),
$$

where $\mu$ is the world index and $\alpha$ is the local coordinate index. The fermionic fields, in the local coordinate system are

$$
\chi^{\alpha}=e_{\mu}^{\alpha} \chi^{\mu}
$$

The Christoffel connection, spin connection and the curvature tensor are defined as

$$
\begin{gathered}
\Gamma_{\nu \lambda}^{\mu}=\frac{1}{2} g^{\mu \sigma}\left(\partial_{\lambda} g_{\sigma \nu}+\partial_{\nu} g_{\sigma \lambda}-\partial_{\sigma} g_{\lambda v}\right), \\
\omega_{\mu, \alpha \beta}=-e_{\alpha \lambda} \partial_{\mu} e_{\beta}^{\lambda}-e_{\alpha \nu} \Gamma_{\mu \lambda}^{\nu} e_{\beta}^{\lambda}, \\
R_{\alpha \beta \gamma \delta}=e_{\alpha}^{\mu} e_{\beta}^{\nu}\left(\partial_{\mu} \omega_{v, \gamma \delta}-\partial_{\nu} \omega_{\mu, \gamma \delta}-\omega_{\mu, \gamma \sigma} \omega_{v, \sigma \delta}+\omega_{v, \gamma \sigma} \omega_{\mu, \sigma \delta}\right) .
\end{gathered}
$$


The action is invariant under global supersymmetry transformation on the world sheet given by

$$
\begin{gathered}
\delta X^{\mu}=\bar{\varepsilon} \chi^{\mu}, \\
\delta \chi^{\alpha}=-i \partial_{\alpha} \gamma^{a} X^{\alpha} \varepsilon-\Gamma_{\nu \varrho}^{\mu} \bar{\varepsilon} \chi^{v} \chi^{\varrho} e_{\mu}^{\alpha} .
\end{gathered}
$$

$\varepsilon$ is a constant Majorana spinor in two dimensions.

The generators of the superconformal transformations on $S^{2}$ are

$$
\begin{gathered}
J_{ \pm}=2 S_{ \pm}^{\alpha} \psi_{ \pm \alpha} \\
T_{ \pm}=S_{ \pm}^{\alpha} S_{ \pm \alpha} \mp \frac{i}{2}\left(D_{\sigma} \psi_{ \pm}^{\alpha}\right) \psi_{ \pm \alpha}+\frac{1}{24} R_{\alpha \beta \gamma \delta}\left[\psi_{+}^{\alpha}, \psi_{-}^{\gamma}\right]\left[\psi_{+}^{\beta}, \psi_{-}^{\delta}\right]
\end{gathered}
$$

where

$$
S_{ \pm \alpha}=\frac{1}{2}\left(P_{\mu} e_{\alpha}^{\mu}+\frac{i}{2} \omega_{\mu, \gamma \delta} \chi^{\gamma} \chi^{\delta} e_{\alpha}^{\mu} \pm X^{\prime \mu} e_{\mu \alpha}\right)
$$

and $\psi_{ \pm \alpha}$ are the two dimensional chiral fermions defined in Sect. II.

The Eqs. (4.9a) and (4.9b) are generalized to $\Sigma$ as follows:

$$
\begin{gathered}
J_{ \pm}(Q)=2 S_{ \pm}^{\alpha}(Q) \psi_{ \pm \alpha}(Q) \\
T_{ \pm}(Q)=S_{ \pm}^{\alpha}(Q) S_{ \pm \alpha}(Q) \mp \frac{i}{2}\left(D_{Q} \psi_{ \pm}^{\alpha}(Q)\right) \psi_{ \pm \alpha}(Q) \\
+\frac{1}{24} R_{\alpha \beta \gamma \delta}\left[\psi_{+}^{\alpha}(Q), \psi_{-}^{\gamma}(Q)\right]\left[\psi_{+}^{\beta}(Q), \psi_{-}^{\delta}(Q)\right] .
\end{gathered}
$$

We can derive the classical superconformal algebra for the generators (4.11), analogous to (3.16)-(3.19) as derived for the free string in Sect. III. The algebra will go over to the algebra on $S^{2}$ once we restrict ourselves to any admissible coordinate patch. However, in order to derive the quantum constraint algebra we have to define normal ordered generators in the quantized theory on $\Sigma$.

We follow the method of Riemann normal coordinate expansion [17] and expand the normal ordered generators of the superconformal transformations to order $R$ as follows:

$$
\begin{gathered}
X^{\mu}=X_{B}^{\mu}+\xi^{\mu}(Q), \\
e_{\mu \alpha}(X(Q))=\eta_{\mu \alpha}-\frac{1}{6} R_{\mu \lambda \alpha \kappa}\left(X_{B}\right) \xi^{\lambda}(Q) \xi^{\kappa}(Q), \\
g_{\mu \nu}(X)=g_{\mu \nu}\left(X_{B}\right)+\frac{1}{3} R_{\mu \lambda v \varrho}\left(X_{B}\right) \xi^{\lambda}(Q) \xi^{\varrho}(Q) .
\end{gathered}
$$

$X_{B}^{\mu}$ is the background field and $\xi^{\mu}(Q)$ is the fluctuation. Henceforth, we suppress $X_{B}^{\mu}$ dependence of all target space tensors for notational convenience.

The operator

$$
\mathbb{U}=1+\mathbb{K}=1+\int d \sigma k(\sigma)
$$

was introduced in [12] in order to facilitate computation of various quantum brackets in an efficient and elegant manner. One of the advantages of the method proposed in [12] was that all the generators in the interacting theory to order $R$ could be obtained easily from the generators of the free string theory through the 
introduction of the operator $\mathbb{U}$; if we choose

$$
k(\sigma)=-\frac{i}{6} R_{\alpha \lambda \beta \kappa}: \xi^{\lambda}(\sigma) \xi^{\kappa}(\sigma) \psi_{+}^{\alpha}(\sigma) \psi_{-}^{\beta}(\sigma): .
$$

The generalization of the generator, $k(\sigma)$, introduced in (4.13) can be defined on $\Sigma$ as

$$
k(Q)=-\frac{i}{6} R_{\alpha \lambda \beta \kappa}: \xi^{\lambda}(Q) \xi^{\kappa}(Q) \psi_{+}^{\alpha}(Q) \psi_{-}^{\beta}(Q):
$$

to obtain generators of the interacting theory to order $R$; in particular

$$
: J_{ \pm}(Q):=: J_{ \pm}^{0}(Q):+i\left[\mathbb{K},: J_{ \pm}^{0}(Q):\right]
$$

where

$$
\mathbb{K}=\underset{Q^{\prime} \in C_{\tau}}{\oint} k\left(Q^{\prime}\right)
$$

and $J_{ \pm}^{0}(Q)$ are the supercharge densities for the free NSR string given by Eq. (3.5b). The explicit form of the normal ordered supercharge densities are

$$
\begin{gathered}
: J_{+}(Q):=: J_{+}^{0}(Q):+: C_{-}(Q): \\
-\frac{i}{6} R_{\alpha \kappa} \oint_{Q^{\prime} \in C_{\tau}}\left[\bar{\Delta}_{\tau}^{+} \bar{\delta}_{\tau}^{+}-\bar{\Delta}_{\tau}^{-} \bar{\delta}_{\tau}^{-}\right]\left(Q^{\prime}, Q\right): \xi^{\alpha}\left(Q^{\prime}\right) \psi_{-}^{\kappa}(Q): \\
: J_{-}(Q):=: J_{-}^{0}(Q):-: C_{+}:+\frac{i}{6} R_{\alpha \kappa} \oint_{Q^{\prime} \in C_{\tau}}\left[\Delta_{\tau}^{+} \delta_{\tau}^{+}-\Delta_{\tau}^{-} \delta_{\tau}^{-}\right]\left(Q^{\prime}, Q\right): \xi^{\alpha}\left(Q^{\prime}\right) \psi_{+}^{\kappa}\left(Q^{\prime}\right):,
\end{gathered}
$$

where

$$
\begin{aligned}
& C_{-}=-\frac{1}{3} \hat{R}_{\mu \lambda \alpha \kappa}\left[P_{-}^{\mu}(Q) \xi^{\lambda}(Q) \xi^{\kappa}(Q) \psi_{+}^{\alpha}(Q)+i \xi^{\kappa}(Q) \psi_{+}^{\mu}(Q) \psi_{-}^{\alpha}(Q) \psi_{-}^{\lambda}(Q)\right], \\
& C_{+}=-\frac{1}{3} \hat{R}_{\mu \lambda \alpha \kappa}\left[P_{+}^{\mu}(Q) \xi^{\lambda}(Q) \xi^{\kappa}(Q) \psi_{-}^{\alpha}(Q)+i \xi^{\kappa}(Q) \psi_{-}^{\mu}(Q) \psi_{+}^{\alpha}(Q) \psi_{+}^{\lambda}(Q)\right],
\end{aligned}
$$

and

$$
\hat{R}_{\mu \lambda \alpha \kappa}=\frac{1}{2}\left[R_{\mu \lambda \alpha \kappa}+R_{\mu \kappa \alpha \lambda}\right] .
$$

The anticommutators of $J_{ \pm}$can be computed in a straightforward manner using (4.15),

$$
\begin{aligned}
\left\{: J_{ \pm}(Q):,: J_{ \pm}\left(Q^{\prime}\right):\right\}=\{: & \left.J_{ \pm}^{0}(Q):,: J_{ \pm}^{0}\left(Q^{\prime}\right):\right\} \\
\pm & i\left\{\left[: \mathbb{K}:,: J_{\mp}^{0}(Q)\right],: J_{ \pm}^{0}\left(Q^{\prime}\right):\right\} \pm i\left\{: J_{ \pm}^{0}(Q):,\left[: \mathbb{K}:, J_{\mp}^{0}\left(Q^{\prime}\right):\right]\right\}, \\
\left\{: J_{+}(Q):,: J_{-}\left(Q^{\prime}\right):\right\}= & i\left\{\left[: \mathbb{K}:, J_{-}^{0}(Q):\right],: J_{-}^{0}\left(Q^{\prime}\right):\right\} \\
& -i\left\{\left[: J_{+}^{0}(Q):,\left[: \mathbb{K}:,: J_{+}^{0}\left(Q^{\prime}\right):\right]\right\} .\right.
\end{aligned}
$$

The anticommutators (4.18) and (4.19) can be evaluated using (4.16) and (4.17). They are presented below

$$
\begin{gathered}
i\left\{\left[: \mathbb{K}:,: J_{-}^{0}(Q):\right],: J_{+}^{0}\left(Q^{\prime}\right)\right\}+i\left\{: J_{+}^{0}(Q):,\left[: \mathbb{K}:,: J_{-}^{0}\left(Q^{\prime}\right):\right]\right\} \\
=: E\left(Q, Q^{\prime}\right):+: V\left(Q, Q^{\prime}\right):+: L\left(Q, Q^{\prime}\right):,
\end{gathered}
$$


where

$$
\begin{aligned}
: E\left(Q, Q^{\prime}\right):= & \frac{2}{3} \hat{R}_{\mu \lambda \alpha \kappa}\left[i: P_{-}^{\mu}(Q) \xi^{\kappa}(Q) \psi_{+}^{\alpha}(Q) \psi_{+}^{\lambda}\left(Q^{\prime}\right): \Delta_{\tau}\left(Q, Q^{\prime}\right)\right. \\
& +: P_{-}^{\mu}(Q) \xi^{\lambda}(Q) \xi^{\kappa}(Q) P_{+}^{\alpha}\left(Q^{\prime}\right): \delta_{\tau}\left(Q, Q^{\prime}\right) \\
& -\frac{1}{2}: \psi_{+}^{\mu}(Q) \psi_{-}^{\alpha}(Q) \psi_{-}^{\lambda}(Q) \psi_{+}^{\kappa}\left(Q^{\prime}\right): \Delta_{\tau}\left(Q, Q^{\prime}\right) \\
& \left.+i: \xi^{\kappa}(Q) \psi_{-}^{\alpha}(Q) \psi_{-}^{\lambda}(Q) P_{+}^{\mu}\left(Q^{\prime}\right): \delta_{\tau}\left(Q, Q^{\prime}\right)\right] \\
& +\frac{1}{6} \hat{R}_{\mu \lambda \alpha \kappa}: \xi^{\lambda}(Q) \xi^{\kappa}(Q): \delta_{\tau}\left(Q, Q^{\prime}\right) \sum_{n, m} \omega_{n}(Q) \bar{\omega}_{m}\left(Q^{\prime}\right)\left\langle\alpha_{n}^{\mu} \bar{\alpha}_{m}^{v}\right\rangle \\
& +\left(Q \leftrightarrow Q^{\prime}\right),
\end{aligned}
$$

$$
\begin{aligned}
: V\left(Q, Q^{\prime}\right):= & -\frac{1}{3} R_{\alpha \kappa}\left[2 i:\left(\Delta_{\tau}^{-} \delta_{\tau}^{-}-\Delta_{\tau}^{+} \delta_{\tau}^{+}\right)\left(Q, Q^{\prime}\right): P_{+}^{\alpha}(Q) \xi^{\kappa}(Q):\right. \\
& -i: \xi^{\kappa}\left(Q^{\prime}\right) P_{+}^{\alpha}\left(Q^{\prime}\right):\left(\bar{\Delta}_{\tau}^{+} \bar{\delta}_{\tau}^{+}-\bar{\Delta}_{\tau}^{-} \bar{\delta}_{\tau}^{-}\right)\left(Q^{\prime}, Q\right) \\
& +\left(Q \leftrightarrow Q^{\prime}\right),
\end{aligned}
$$

$$
\begin{aligned}
L\left(Q, Q^{\prime}\right)= & -\frac{1}{6} R \underset{Q^{\prime \prime} \in C_{\tau}}{\oint}\left(\bar{\Delta}_{\tau}^{+} \bar{\delta}_{\tau}^{+}-\bar{\Delta}_{\tau}^{-} \bar{\delta}_{\tau}^{-}\right)\left(Q^{\prime \prime}, Q\right)\left(\Delta_{\tau}^{-} \delta_{\tau}^{-}-\Delta_{\tau}^{+} \delta_{\tau}^{+}\right)\left(Q^{\prime \prime}, Q^{\prime}\right) \\
& +\left(Q \leftrightarrow Q^{\prime}\right) .
\end{aligned}
$$

Thus

$$
\begin{aligned}
\left\{: J_{+}(Q):,: J_{+}\left(Q^{\prime}\right):\right\}= & \left\{: J_{+}^{0}(Q):,: J_{+}^{0}\left(Q^{\prime}\right):\right\}+: E\left(Q, Q^{\prime}\right): \\
& +: V\left(Q, Q^{\prime}\right):+L\left(Q, Q^{\prime}\right)
\end{aligned}
$$

We recall that the first term on the right-hand side is given by (3.55).

If we choose a local coordinate system, $W=\tau+i \sigma=\ln z$ and $W^{\prime}=\tau+i \sigma^{\prime}=\ln z^{\prime}$ Eqs. (4.21) (4.23) take the following form (see Appendix A):

$$
\begin{aligned}
& E\left(Q, Q^{\prime}\right) \rightarrow \frac{4}{3} \hat{R}_{\mu \lambda \alpha \kappa}\left[: P_{+}^{\alpha}(\sigma) \xi^{\lambda}(\sigma) \xi^{\kappa}(\sigma) P_{-}^{\mu}(\sigma):\right. \\
& \quad-i: P_{-}^{\mu}(\sigma) \xi^{\kappa}(\sigma) \psi_{+}^{\lambda}(\sigma) \psi_{+}^{\alpha}(\sigma): \\
& \quad-i: P_{+}^{\mu}(\sigma) \xi^{\kappa}(\sigma) \psi_{-}^{\lambda}(\sigma) \psi_{-}^{\alpha}(\sigma): \\
& \left.\quad+\frac{1}{2}: \psi_{+}^{\mu}(\sigma) \psi_{-}^{\alpha}(\sigma) \psi_{+}^{\kappa}(\sigma) \psi_{-}^{\lambda}(\sigma):\right] \delta\left(\sigma-\sigma^{\prime}\right), \\
& V\left(Q, Q^{\prime}\right) \rightarrow \frac{1}{6 \pi} R_{\alpha \kappa} \frac{\partial}{\partial \sigma}\left[\xi^{\kappa}(\sigma) \xi^{\prime \alpha}(\sigma)\right] \delta\left(\sigma-\sigma^{\prime}\right), \\
& L\left(Q, Q^{\prime}\right) \rightarrow \frac{1}{2 \pi} R \delta^{\prime \prime}\left(\sigma-\sigma^{\prime}\right) .
\end{aligned}
$$

The local form of $T_{+}^{0}(Q)$ obtained in Sect. III together with the expressions for $E$, $V$, and $L$ in the local form (4.25) can be used to obtain a local form of $T_{+}(\sigma)$ on $S^{2}$. This local form can be generalized to $\Sigma$ and subsequently we compute the algebras involving $T_{+}(Q)$. We recall

$$
4: T_{+}(\sigma):=4: T_{+}^{0}(\sigma):+: E(\sigma):+: V(\sigma):+: L(\sigma):,
$$

which has the corresponding form on $\Sigma$ as

$$
4: T_{+}(Q):=4: T_{+}^{0}(Q):+: E(Q):+: V(Q):: L(Q): .
$$


The commutator of $J_{+}$and $J_{-}$has the following form:

$$
\begin{aligned}
\left\{: J_{+}(Q):,: J_{-}\left(Q^{\prime}\right):\right\}= & R_{\alpha \beta}\left[\frac{1}{2}: \psi_{+}^{\alpha}(Q) \psi_{-}^{\beta}(Q):\left(\bar{\Delta}_{\tau}^{+} \bar{\delta}_{\tau}^{+}-\bar{\Delta}_{\tau}^{-} \bar{\delta}_{\tau}^{-}\right)\left(Q, Q^{\prime}\right)\right. \\
& -\frac{1}{2}: \psi_{+}^{\alpha}\left(Q^{\prime}\right) \psi_{-}^{\beta}\left(Q^{\prime}\right):\left(\Delta_{\tau}^{+} \delta_{\tau}^{+}-\Delta_{\tau}^{-} \delta_{\tau}^{-}\right)\left(Q^{\prime}, Q\right) \\
& +\frac{1}{6}: \psi_{+}^{\alpha}(Q) \psi_{-}^{\beta}(Q):\left(\Delta_{\tau}^{+} \delta_{\tau}^{+}-\Delta_{\tau}^{-} \delta_{\tau}^{-}\right)\left(Q, Q^{\prime}\right) \\
& \left.-\frac{1}{6}: \psi_{+}^{\alpha}\left(Q^{\prime}\right) \psi_{-}^{\beta}\left(Q^{\prime}\right):\left(\bar{\Delta}_{\tau}^{+} \bar{\delta}_{\tau}^{+}-\bar{\Delta}_{\tau}^{-} \bar{\delta}_{\tau}^{-}\right)\left(Q^{\prime}, Q\right)\right] \\
& +\hat{R}_{\mu \lambda \alpha \kappa} S^{\mu \lambda \alpha \kappa} .
\end{aligned}
$$

Notice the last term on the right-hand side of (4.28) has a tensor $S^{\mu \lambda \alpha \kappa}\left(Q, Q^{\prime}\right)$ contracted with $\hat{R}_{\mu \lambda \alpha \kappa}$. This fourth rank tensor in space-time manifold is also a tensor on $\Sigma$ of weight $3 / 2$ in $Q$ as well as of the same weight in $Q^{\prime}$. However, the last term of (4.28) vanishes when evaluated in a local coordinate system (see Appendix A for further details). Thus it is dropped from (4.28). We also drop all such terms from all the expressions in our future computations.

Now we proceed to evaluate the rest of the algebra involving $T_{+}(Q)$ given by (4.27),

$$
\begin{aligned}
& {\left[: T_{+}(Q):,: J_{+}\left(Q^{\prime}\right):\right]=\left[: T_{+}^{0}(Q):,: J_{+}^{0}\left(Q^{\prime}\right):\right]} \\
& \quad+R_{\alpha \kappa}\left(\frac{1}{6}: P_{+}^{\kappa}(Q) \psi_{+}^{\alpha}(Q):\left[\bar{\Delta}_{\tau}^{+} \bar{\delta}_{\tau}^{+}-\bar{\Delta}_{\tau}^{-} \bar{\delta}_{\tau}^{-}\right]\left(Q, Q^{\prime}\right)\right. \\
& \quad+\frac{1}{6}: \xi^{\kappa}(Q) d_{Q} \psi_{+}^{\alpha}(Q):\left[\bar{\Delta}_{\tau}^{+} \bar{\delta}_{\tau}^{+}-\bar{\Delta}_{\tau}^{-} \bar{\delta}_{\tau}^{-}\right]\left(Q, Q^{\prime}\right) \\
& \quad-\frac{1}{12} d_{Q}\left\{\left[\bar{\Delta}_{\tau}^{+} \bar{\delta}_{\tau}^{+}-\bar{\Delta}_{\tau}^{-} \bar{\delta}_{\tau}^{-}\right]\left(Q, Q^{\prime}\right): \xi^{\kappa}(Q) \psi_{+}^{\alpha}(Q):\right\} \\
& \quad+\frac{1}{2}: P_{-}^{\alpha}(Q) \psi_{+}^{\kappa}(Q):\left[\Delta_{\tau}^{+} \delta_{\tau}^{+}-\Delta_{\tau}^{-} \delta_{\tau}^{-}\right]\left(Q, Q^{\prime}\right) \\
& \quad+\frac{i}{24 \pi} d_{Q^{\prime}} d_{Q^{\prime}}\left\{\Delta_{\tau}\left(Q, Q^{\prime}\right): \xi^{\alpha}(Q) \psi_{+}^{\beta}\left(Q^{\prime}\right):\right\} \\
& \left.\quad-\frac{1}{6}: P_{-}^{\alpha}(Q) \psi_{+}^{\kappa}\left(Q^{\prime}\right):\left[\Delta_{\tau}^{+} \delta_{\tau}^{+}-\Delta_{\tau}^{-} \delta_{\tau}^{-}\right]\left(Q^{\prime}, Q\right)\right)
\end{aligned}
$$

We may remark that this expression goes over to the corresponding expressions on $S^{2}$ upon restriction to a suitable local coordinate system.

$$
\begin{aligned}
{\left[: T_{+}(Q):: J_{-}\left(Q^{\prime}\right):\right]=} & R_{\alpha \kappa}\left(\frac{1}{6}: P_{+}^{\kappa}(Q) \psi_{-}^{\alpha}\left(Q^{\prime}\right):\left[\Delta_{\tau}^{+} \delta_{\tau}^{+}-\Delta_{\tau}^{-} \delta_{\tau}^{-}\right]\left(Q, Q^{\prime}\right)\right. \\
& +\frac{1}{6}: \xi^{\kappa}\left(Q^{\prime}\right) \psi_{-}^{\alpha}\left(Q^{\prime}\right):\left[\delta_{\tau}^{+} d_{Q^{\prime}} \delta_{\tau}^{+}-\delta_{\tau}^{-} d_{Q_{\tau}} \delta_{\tau}^{-}\right]\left(Q^{\prime}, Q\right) \\
& -\frac{1}{6}: P_{+}^{\alpha}\left(Q^{\prime}\right) \psi_{-}^{\alpha}\left(Q^{\prime}\right):\left[\Delta_{\tau}^{+} \delta_{\tau}^{+}-\Delta_{\tau}^{-} \delta_{\tau}^{-}\right]\left(Q^{\prime}, Q\right) \\
& -\frac{1}{2}: P_{+}^{\alpha}(Q) \psi_{-}^{\kappa}(Q):\left[\bar{\Delta}_{\tau}^{-} \bar{\delta}_{\tau}^{-}-\bar{\Delta}_{\tau}^{+} \bar{\delta}_{\tau}^{+}\right]\left(Q, Q^{\prime}\right) \\
& +\frac{i}{24 \pi} d_{Q} d_{Q}\left[: \xi^{\alpha}(Q) \psi_{-}^{\kappa}\left(Q^{\prime}\right): \Delta_{\tau}\left(Q, Q^{\prime}\right)\right] \\
& -\frac{i}{12 \pi}: \xi^{\kappa}\left(Q^{\prime}\right) \Psi_{-}^{\alpha}\left(Q^{\prime}\right): \sum_{n, m}\left[\omega_{n}(Q) \omega_{m}\left(Q^{\prime}\right) \tilde{\gamma}_{n m}\right. \\
& \left.\left.\times \Delta_{\tau}^{+}\left(Q^{\prime}, Q\right)+\omega_{m}(Q) \omega_{n}\left(Q^{\prime}\right) \tilde{\gamma}_{n m} \Delta_{\tau}^{-}\left(Q^{\prime}, Q\right)\right]\right) .
\end{aligned}
$$

The commutators $\left[T_{+}(Q), T_{ \pm}\left(Q^{\prime}\right)\right]$ can be computed in a straightforward manner following the procedures stated above. In fact we have carried out explicit calculations for these brackets. However, the expressions are very lengthy when $Q$ and $Q^{\prime}$ are taken to be two arbitrary points on $\Sigma$. They are reduced to simple forms 
when we evaluate them on a local coordinate system. This form exactly matches with the quantum algebra of $S^{2}$ as expected. In what follows, we present the results of the two commutators in a local coordinate system.

$$
\begin{aligned}
& {\left[: T_{+}(\sigma):,: T_{+}\left(\sigma^{\prime}\right):\right]} \\
& =\left[Z(\sigma)+i: T_{+}^{0}(\sigma):+Z\left(\sigma^{\prime}\right)+i: T_{+}^{0}\left(\sigma^{\prime}\right):\right] \frac{\partial}{\partial \sigma} \delta\left(\sigma-\sigma^{\prime}\right) \\
& -\frac{i}{2 \pi}\left(D-\frac{R}{6 \pi}\right) \frac{\partial^{3}}{\partial \sigma^{3}} \delta\left(\sigma-\sigma^{\prime}\right), \\
& {\left[T_{+}(\sigma), T_{-}\left(\sigma^{\prime}\right)\right]=2 \frac{\partial}{\partial \sigma} Z(\sigma) \delta\left(\sigma-\sigma^{\prime}\right),}
\end{aligned}
$$

where

$$
Z(\sigma)=-\frac{i}{6 \pi} R_{\alpha \beta}: P_{+}^{\alpha}(\sigma) P_{-}^{\beta}(\sigma):
$$

We observe that the superconformal algebra, in the case of the interacting theory, contains operator anomalies in addition to the usual $C$-number anomaly terms. Furthermore, the central charge term in the commutator of the energy momentum tensor has an additional piece proportional to $R$ the scalar curvature of the target manifold. We may mention that the theory is free from all anomalies if the space-time dimension, $D=10$ and the manifold is Ricci flat, i.e. $R_{\mu \nu}=0$.

In order to derive the condition for vanishing of these anomalies, we reduce the bilocal form of the constraint algebra on $\Sigma$ to a local form by considering an admissible local coordinate system although the algebra is derived for two operators defined at generic points $Q$ and $Q^{\prime}$ on $\Sigma$ (see Eqs. (4.24), (4.28), (4.29), and (4.30)). We also observe that the quantum brackets of "holomorphic" and "antiholomorphic" generators such as $T_{+}(Q)$ with $T_{-}\left(Q^{\prime}\right), J_{-}\left(Q^{\prime}\right)$ and $J_{+}$with $T_{-}$, $J_{-}$do not vanish as is the case for the free string. In fact these brackets are proportional to the Ricci tensor; however, these anomalies vanish for the Ricci flat manifold as noted earlier.

\section{Summary and Conclusions}

We have investigated the evolution of NSR superstring for a world sheet of arbitrary topology, and examined the consequences of (super)conformal invariance. The Fubini-Veneziano fields are defined on a Riemann surface $\Sigma$ in the KN global operator formalism. Then the generators of the superconformal transformations were expanded in the KN global bases and the superconformal algebra, on $\Sigma$, for both the free as well as the interacting superstring was derived.

All the anomalies were obtained from the algebra of the generators in the quantized theory. It was shown that the operator and the $c$-number anomalies vanish if the space-time dimension is ten and the target manifold is Ricci flat. We note that our results are manifestly covariant as we have used the Riemann normal coordinate expansion method in defining all our generators in the quantum theory. It will be interesting to examine the higher order corrections (in $R$ ) to the background field equations of motion when next to leading order terms (in normal coordinate expansions) are taken into consideration. 
We remark that our results can be derived from the nilpotency of the quantum BRST charge defined on $\Sigma$. In computing the anticommutator of the normal ordered BRST charge one has to essentially derive the full superconformal as was done in [18] for the NSR string in general background (in the case of $S^{2}$ ). Thus, the results presented here can be deduced from the requirement of the nilpotency of the BRST charge.

In conclusion, the $\mathrm{KN}$ global operator formalism has proved to be an elegant procedure to probe the consequences of superconformal invariance properties of superstring theories on world sheet of arbitrary topology.

\section{Appendix A}

In this appendix we briefly outline the procedure for reducing any global expression on $\Sigma$ to a local coordinate system defined as $W(Q)=\tau(Q)+i \sigma(Q)$. The procedure is the following: locally on $C_{\tau}$ we have

$$
\begin{aligned}
& d_{Q}=d W \frac{\partial}{\partial \sigma}=d \sigma_{M} \frac{\partial}{\partial \sigma_{M}}, \\
& d W=d \sigma_{M} \frac{\partial}{\partial \sigma_{M}}=\frac{\partial}{\partial \sigma_{M}},
\end{aligned}
$$

where $M$ stands for the Minkowski signature and the world sheet is parametrized by $(\tau, \sigma)$.

The global bases can be written in the local coordinate system $W$; in particular we have all expressions in $z_{ \pm}$coordinates as given in Eqs. (2.3)-(2.7). It was stated in Sect. II that $C_{\tau}$ reduces to the circle $C_{ \pm}$around $P_{ \pm}$as $\tau \rightarrow \mp \infty$. We denote this system as $z_{+}$,

$$
W=\tau+i \sigma=\ln z, \quad z=\exp (\tau+i \sigma) .
$$

Now, if $f(Q)$ is a smooth one form on $C_{\tau}$, we have the defining relations (Table 1 )

$$
\oint_{\mathcal{C}_{\tau}} f(Q) \Delta_{\tau}\left(Q, Q^{\prime}\right)=f\left(Q^{\prime}\right)
$$

as (A.3) is valid on any $C_{\tau}$ in particular for $C_{ \pm}$(in the local coordinate system given by (A.2))

$$
\Delta_{\tau}\left(Q, Q^{\prime}\right)=\delta\left(\sigma-\sigma^{\prime}\right) d W^{\prime}
$$

Note that $\delta\left(\sigma-\sigma^{\prime}\right)$ is the Minkowski space $\delta$-function and this convention is followed everywhere else.

Similarly,

$$
\delta_{\tau}\left(Q, Q^{\prime}\right)=\delta\left(\sigma-\sigma^{\prime}\right)(d W)^{1 / 2}\left(d W^{\prime}\right)^{1 / 2} .
$$

For the other distributions which appear in (3.49)-(3.51) we have the following expressions in the $W$ coordinate system:

$$
\begin{aligned}
& \Delta_{\tau}^{+}\left(Q^{\prime}, Q\right)=\bar{\Delta}_{\tau}^{+}\left(Q^{\prime}, Q\right)=\frac{1}{2 \pi i} \frac{1}{\sigma-\sigma^{\prime}-i \varepsilon} d W, \\
& \Delta_{\tau}\left(Q^{\prime}, Q\right)=\bar{\Delta}_{\tau}^{-}\left(Q^{\prime}, Q\right)=-\frac{1}{2 \pi i} \frac{1}{\sigma-\sigma^{\prime}+i \varepsilon} d W,
\end{aligned}
$$




$$
\begin{gathered}
\bar{\delta}_{\tau}^{+}\left(Q^{\prime}, Q\right)=\bar{\delta}_{\tau}^{+}\left(Q^{\prime}, Q\right)=\frac{1}{2 \pi i} \frac{1}{\sigma-\sigma^{\prime}-i \varepsilon}(d W)^{1 / 2}\left(d W^{\prime}\right)^{1 / 2}, \\
\delta_{\tau}^{-}\left(Q^{\prime}, Q\right)=\bar{\delta}^{-}\left(Q^{\prime}, Q\right)=-\frac{1}{2 \pi i} \frac{1}{\sigma-\sigma^{\prime}+i \varepsilon}(d W)^{1 / 2}\left(d W^{\prime}\right)^{1 / 2},
\end{gathered}
$$

where

$$
\delta\left(\sigma-\sigma^{\prime}\right)=\frac{1}{2 \pi i}\left[\frac{1}{\sigma-\sigma^{\prime}-i \varepsilon}-\frac{1}{\sigma-\sigma^{\prime}+i \varepsilon}\right]
$$

with

$$
d W=-d \bar{W}, \quad(d W)^{1 / 2}=i(d \bar{W})^{1 / 2} .
$$

It follows from (A.5)-(A.7),

$$
\begin{aligned}
& {\left[\Delta_{\tau}^{+} \delta_{\tau}^{+}-\Delta_{\tau}^{-} \delta_{\tau}^{-}\right]\left(Q^{\prime}, Q\right)=-\frac{1}{2 \pi i} \frac{\partial}{\partial \sigma} \delta\left(\sigma-\sigma^{\prime}\right)(d W)^{3 / 2}\left(d W^{\prime}\right)^{1 / 2},} \\
& {\left[\bar{\Delta}_{\tau}^{+} \bar{\delta}_{\tau}^{+}-\bar{\Delta}_{\tau}^{-} \bar{\delta}_{\tau}^{-}\right]\left(Q^{\prime}, Q\right)=-\frac{1}{2 \pi i} \frac{\partial}{\partial \sigma}, \delta\left(\sigma-\sigma^{\prime}\right)(d W)^{3 / 2}(d W)^{1 / 2} .}
\end{aligned}
$$

We can evaluate other combinations of such delta-tensors in a similar fashion. The fundamental fields $X^{\mu}(Q), P^{\mu}(Q), \psi_{ \pm}^{\mu}(Q)$, and $P_{ \pm}^{\mu}(Q)$ take the following form on the coordinate system (A.2):

$$
\begin{gathered}
X^{\mu}(Q) \equiv X^{\mu}(\sigma), \\
P^{\mu}(Q) \equiv P^{\mu}(\sigma) d W, \\
\psi_{ \pm}^{\mu}(Q) \equiv \psi_{ \pm}^{\mu}(\sigma)(d W)^{1 / 2}, \\
P_{ \pm}^{\mu}(Q) \equiv P_{ \pm}^{\mu}(\sigma) d W .
\end{gathered}
$$

Using these results it is easy to check that the constraint algebras on $\Sigma$ reduce to that on the sphere in the coordinate system (A.2); recall Eq. (3.20).

Let us focus our attention on the last term of (4.28), where the anticommutator of $J_{+}$and $J_{-}$is computed. As the algebra is defined on an equal time curve $C_{\tau}$, any term which vanishes on one such curve will vanish on all others. This is a consequence of the duality and the completeness relations which hold on any $C_{\tau}$. If we write $\widehat{R}_{\mu \lambda \alpha \kappa} S^{\mu \lambda \alpha \kappa}$ on any equal time curve, $C_{\tau}$, in the coordinate patch defined by (A.2); then $C_{\tau}$ becomes the circle $C_{+}$around $P_{+}$. The delta-tensors and the fundamental fields along with their derivatives go over to the expressions (A.1)-(A.7) and (A.9). It turns out that this term vanishes when we use the properties of the standard Dirac $\delta$-function and the symmetry properties of $\hat{R}_{\mu \lambda \alpha \kappa}$ and other tensors that appear in the product. Thus this term vanishes on every $C_{\tau}$ on $\Sigma$. The same argument goes through for similar expressions that appear in computation of various brackets. As mentioned in the text, we suppress appearance of such terms in the algebra.

\section{Appendix B}

We discuss the cancellation of the $c$-number anomaly in the algebra $\left[T_{+}^{0}(Q)\right.$, $\left.T_{+}^{0}\left(Q^{\prime}\right)\right]$ which appears when we consider the quantum operators (see 3.57). The full 
gauge fixed action gets contributions from the $(b, c)$ and the $(\beta, \gamma)$ ghost systems. The ghost part of the energy momentum tensor on $\Sigma$ has the following form [9]:

$$
T^{g h}(Q)=c(Q) d_{Q} b(Q)+2 d_{Q} c(Q) b(Q)-\frac{1}{2} \gamma(Q) d_{Q} \beta(Q)-\frac{3}{2} d_{Q} \gamma(Q) \beta(Q) .
$$

These fields are meromorphic forms of weight $\lambda$ with the following Laurent expansion (see Table 1):

$$
\begin{gathered}
c(Q)=\sum_{k} c_{k} e_{k}(Q), \quad \lambda=-1, \\
b(Q)=\sum_{k} b_{k} \Omega_{k}(Q), \quad \lambda=2, \\
\gamma(Q)=\sum_{\alpha} \gamma_{\alpha} g_{\alpha}(Q), \quad \lambda=-1 / 2, \\
\beta(Q)=\sum_{\alpha} \beta_{\alpha} k_{\alpha}(Q) \lambda=3 / 2 .
\end{gathered}
$$

The fundamental commutators and anticommutators are

$$
\begin{aligned}
& \left\{c(Q), b\left(Q^{\prime}\right)\right\}=i D_{\tau}\left(Q, Q^{\prime}\right), \\
& {\left[\gamma(Q), \beta\left(Q^{\prime}\right)\right]=i d_{\tau}\left(Q, Q^{\prime}\right) .}
\end{aligned}
$$

The correlators involving $(b, c)$ and $(\beta, \gamma)$ ghost fields are as follows:

$$
\begin{aligned}
\left\langle c\left(Q^{\prime}\right) b(Q)\right\rangle & =\frac{1}{2 \pi} \sum_{k<g_{0}} e_{k}\left(Q^{\prime}\right) \Omega_{k}(Q), \\
\left\langle b\left(Q^{\prime}\right) c(Q)\right\rangle & =\frac{1}{2 \pi} \sum_{k \geqq g_{0}} e_{k}(Q) \Omega_{k}\left(Q^{\prime}\right), \\
\left\langle\gamma\left(Q^{\prime}\right) \beta(Q)\right\rangle & =\frac{1}{2 \pi} \sum_{\alpha<g} g_{\alpha}\left(Q^{\prime}\right) k_{\alpha}(Q), \\
\left\langle\beta\left(Q^{\prime}\right) \gamma(Q)\right\rangle & =-\frac{1}{2 \pi} \sum_{\alpha \geqq g} g_{\alpha}(Q) k_{\alpha}\left(Q^{\prime}\right) .
\end{aligned}
$$

Using the correlators (B.4) along with the duality and the completeness relations for the bases in Table 1, the central term in $\left[T_{+}^{g h}(Q), T_{+}^{g h}\left(Q^{\prime}\right)\right]$ is given by

$$
\frac{1}{4 \pi^{2}} \sum_{n, m}\left[\tilde{\chi}_{n m}+\chi_{n m}^{\gamma}\right] \omega_{n}(Q) \omega_{m}\left(Q^{\prime}\right)
$$

with

$$
\begin{aligned}
\tilde{\chi}_{n m}= & \sum_{k<g_{0}} \sum_{r, s=-g_{0}}^{+g_{0}} \sum_{n, m} C_{n, k}^{s} C_{m, n+k-s}^{r} \theta_{+}\left(n+k-s-g_{0}\right) \\
& -\sum_{k \geqq g_{0}} \sum_{r, s=-g_{0}}^{+g_{0}} \sum_{\substack{n, m \\
n+m=r+s}}^{r+m} C_{n, k}^{s} C_{m, n+k-s}^{r} \theta_{-}\left(n+k-s-g_{0}\right), \\
\chi_{n m}^{\gamma}= & -\sum_{\alpha<g} \sum_{r, s=-g_{0}}^{+g_{0}} \sum_{\substack{n, m \\
n+m=k+s}} H_{n, \alpha}^{s} H_{m, n+\alpha-s}^{r} \theta_{+}(n+\alpha-s-g) \\
& +\sum_{\alpha \geqq g} \sum_{r, s=-g_{0}}^{+g_{0}} \sum_{\substack{n, m \\
n+m=r+s}} H_{n, \alpha}^{s} H_{m, n+\alpha-s}^{r} \theta_{-}(n+\alpha-s-g),
\end{aligned}
$$


where $C_{i j}^{s}$ and $H_{n \alpha}^{s}$ are given by (2.11) and (2.15). We recall that

$$
\theta_{+}(n)=\left\{\begin{array}{ll}
0 & n<0 \\
1 & n \geqq 0
\end{array} \text { and } \theta_{-}(n)= \begin{cases}0 & n \geqq 0 \\
1 & n<0\end{cases}\right.
$$

The central extension of $\left[T_{+}^{0}(Q), T_{+}^{0}\left(Q^{\prime}\right)\right]$ in (3.57) can be written, in a similar fashion as

$$
\frac{D}{4 \pi^{2}} \sum_{n, m}\left[\chi_{n m}^{\Sigma}+\chi_{n m}^{\psi}\right] \Omega_{n}(Q) \Omega_{m}\left(Q^{\prime}\right)
$$

where

$$
\chi_{n m}^{\psi}=\frac{1}{8} \sum_{\substack{k>0 \\ s<0}}\left[F_{k,-s}^{n} F_{s, k}^{m}-F_{k,-s}^{m} F_{s,-k}^{n}\right]
$$

and

$$
F_{k s}^{n}=\frac{1}{2 \pi i} \oint_{C_{\tau}}\left[h_{k} d h_{s}-h_{s} d h_{k}\right] e_{n}(Q)
$$

and $\chi_{n m}^{\Sigma}$ is as in (2.37) of reference [7].

Hence the central term in $\left[T_{+}^{t}(Q), T_{+}^{t}\left(Q^{\prime}\right)\right]$ is given by $\frac{1}{4 \pi^{2}} \sum_{n, m} \hat{\chi}_{n m} \omega_{n}(Q) \omega_{m}\left(Q^{\prime}\right)$,

$$
\hat{\chi}_{n m}=D\left[\chi_{n m}^{\Sigma}+\chi_{n m}^{\psi}\right]+\tilde{\chi}_{n m}+\chi_{n m}^{\gamma},
$$

where $T_{+}^{t}$ is the full energy momentum tensor, $T_{+}^{t}=T_{+}^{0}+T_{+}^{g h}, \tilde{\chi}_{n m}, \chi_{n m}^{\gamma}, \chi_{n m}^{\Sigma}$, and $X_{n m}^{\psi}$ are as defined in (B.5) and (B.6). The central terms in (B.5)-(B.7) are similar to the expressions in [9]. Note that we have worked in the NS sector.

The central extension is antisymmetric and satisfies a locality condition [7]. Therefore, up to trivial cocycle, it is proportional to the cocycle $X\left(e_{i}, e_{j}\right)$. Using the well known properties of $\chi\left(e_{i}, e_{j}\right)$ we get $\hat{\chi}_{i j}=\left(\frac{3}{2} D-15\right) \chi\left(e_{i}, e_{j}\right)$ up to trivial cocycles. Thus, we conclude that $\hat{\chi}_{i j}=0$ for $D=10$.

Similarly, we can calculate $\hat{\phi}_{\alpha \beta}$ in the algebra for $J_{+}^{0}(Q)$. We note that $\hat{\phi}_{\alpha \beta}$ is symmetric and satisfies locality condition [9]. We then derive the same result as obtained in [9]. This completes our discussion of the critical dimensions and the mechanism of anomaly cancellations.

Acknowledgements. We are thankful to Prof. Ashoke Sen for very valuable discussions. One of us (S.K.P.) is grateful to Profs. R. Sasaki and R. Kubo for many fruitful discussions during his stay at the RITP, Hiroshima. He would like to thank the Institute of Physics. Bhubaneswar for providing a visiting position while this work was initiated and he expresses his gratitude to Prof. C.K. Majumdar for encouragement and support. We would like to thank Prof. S.C. Phatak, Dileep Jatkar and Vijay Agrawal for their help in preparation of this manuscript.

\section{References}

1. Green, M.B., Schwarz, J.H., Witten, E.: Superstring theory Vols. I, II. Cambridge: Cambridge University Press 1987

2. For a review see D'Hoker, E., Phong, D.H.: Rev. Mod. Phys. 60, 917 (1988)

3. Fradkin, E.S., Tseytlin, A.A.: Nucl. Phys. B 261, 1 (1985)

Maharana, J., Veneziano, G.: Nucl. Phys. B 283, 126 (1987) 
4. There is a vast literature on this subject. An up-to-date reference can be found in review articles:

Sen, A.: In: Superstrings, Unified Theories, and Cosmology (Proc. of the Summer Workshop in High Energy Physics and Cosmology, Trieste, 1986), Furlan, G., Jengo, R., Pati, J.C., Sciama, D.W., Shafi, Q. (eds.). Singapore: World Scientific

Callan, C.G., Thorlacius, L.: SLAC Preprint 1989

Tseytlin, A.A.: Int. J. Mod. Phys. A 4, 1257 (1989)

5. Alvarez-Gaumé, L., Gomez, C., Reina, C.: In: Superstrings 87 (Proc. of the Trieste Spring School, 1987), Alvarez-Gaumé, L., Green, M.B., Grisaru, M.T., Iengo, R., Sezgin, E. (eds.). Singapore: World Scientific 1987

Vafa, C.: Phys. Lett. 190B, 47 (1986); 190B, 195 (1986)

Alvarez-Gaumé, L., Gomez, C., Moore, G., Vafa, C.: Nucl. Phys. B 303, 455 (1988)

Alvarez-Gaumé, L., Gomez, C., Nelson, P., Sierra, G., Vafa, C.: Preprint, CERN-TH 5018/1988

6. Witten, E.: Commun. Math. Phys. 113, 529 (1988)

7. Krichever, I.M., Novikov, S.P.: Funk. An. i. Pril. 21 (2), 46 (1987) and 21 (4), 47 (1987)

8. Alberti, J., Taormina, A., Van Baal, P.: Commun. Math. Phys. 120, 249 (1988)

Cotta-Ramusino, P., Martellini, M., Mintchev, M.: Phys. Lett. B 215, 331 (1988)

Bonora, L., Matone, M., Rinaldi, M.: Phys. Lett. B 216, 312 (1989)

Kubo, R., Ojima, S., Paul, S.K.: Mod. Phys. Lett. A 4, 1423 (1989)

9. Bonora, L., Bregola, M., Cotta-Ramusino, P., Martellini, M.: Phys. Lett. B 206, 53 (1988)

Bonora, L., Martellini, M., Rinaldi, M., Russo, J.: Phys. Lett. B 206, 444 (1988)

Bonora, L., Rinaldi, M., Russo, J., Wu, K.: Phys. Lett. B 208, 441 (1988)

Bonora, L., Martellini, M., Rinaldi, M., Wu, K.: Phys. Lett. B 218, 153 (1989)

Lugo, A., Russo, J.: Nucl. Phys. B 322, 210 (1989)

Russo, J.: Nucl. Phys. B 322, 471 (1989)

10. Konisi, G., Saito, T., Takahasi, W.: Prog. Th. Phys. 82, 813 (1989)

11. Saito, T., Wu, K.: Phys. Lett. B 220, 93 (1989)

Osipov, A.A.: Phys. Lett. B 225, 83 (1989)

Kashaev, R.M., Osipov, A.A.: Phys. Lett. B 222, 391 (1989)

Lee, T., Vishwanathan, K.S.: Int. J. Mod. Phys. A 4, 4469 (1989)

Huang, C.S., Zhao, Z.Y.: Phys. Lett. B 220, 87 (1989)

Kubo, R., Yoshi, H., Ojima, S., Paul, S.K.: Prog. Theor. Phys. 82, 1188 (1989)

12. Fubini, S., Maharana, J., Roncadelli, M., Veneziano, G.: Nucl. Phys. B 316, 36 (1989)

13. Das, A., Maharana, J., Roy, S.: FERMILAB-PUB.-89. Nucl. Phys. B (in press)

14. Schlichenmaier, M.: An introduction to Riemann surfaces, algebraic curves, and moduli spaces. Lecture Notes in Mathematics, Vol. 322. Berlin, Heidelberg, New York: Springer 1989

15. Farkas, H., Kra, I.: Riemann surfaces. Berlin, Heidelberg, New York: Springer 1980

16. Bonora, L., Lugo, A., Matone, M., Russo, J.: Commun. Math. Phys. 123, 329 (1989)

17. Alvarez-Gaumé, L., Freedman, D.Z., Mukhi, S.: Ann. Phys. 134, 85 (1983)

18. Das, A., Maharana, J., Roy, S.: Phys. Rev. D 40, 4037 (1989)

Communicated by K. Gawedzki 
